\title{
Experimental Study and Simulation of Film Tension Measurement by Apex Method
}

\author{
Jing Xuanxiang ${ }^{*}$, Xu Zhihong \\ School of Science, Nanjing University of Science and Technology, Nanjing, China

\section{Email address:} \\ 786668311@qq.com (Jing Xuanxiang),xuzh@njust.edu.cn (Xu Zhihong) \\ ${ }^{*}$ Corresponding author
}

\section{To cite this article:}

Jing Xuanxiang, Xu Zhihong. Experimental Study and Simulation of Film Tension Measurement by Apex Method. Science Discovery. Vol. 7, No. 6, 2019, pp. 441-445. doi: 10.11648/j.sd.20190706.21

Received: November 1, 2019; Accepted: December 23 2019; Published: December 26, 2019

\begin{abstract}
In order to ensure the normal use of the tensioned membrane structure, it is necessary to effectively detect the tension applied to the tensioned membrane surface. In this paper, a top-projection membrane surface tension measuring instrument with fixed boundary was designed. During the measure process, the force and the apex displacement were measured simultaneously to obtain the calibration curve group of force and abutment displacement. Compared with the traditional apex method, the measuring instrument eliminates the influence of the membrane structure boundary on the measurement results, and the measurement results were accurate and reliable. The correctness of the device is verified by numerical simulation and experimental results. Theoretically solved the technical problem of rapid measurement of membrane surface tension, and obtained stable results in actual measurement, thus providing a new method for membrane surface tension measurement.
\end{abstract}

Keywords: Top Protrusion Method, Membrane Structure, Membrane Tension Meter

\section{顶突法测膜面张力的实验研究与模拟}

景宣翔", 徐志洪

南京理工大学理学院, 南京, 中国

邮箱

786668311@qq.com（景宣翔）, xuzh@njust.edu.cn（徐志洪）

摘要: 为了保证张拉膜结构的正常使用, 需要对张拉膜面所受的张力进行有效的检测。该文设计了一种具有固定边界的顶 突式膜面张力测量仪, 在测量时, 同时测量力与顶突位移, 得到力与顶突位移的标定曲线族。与传统的顶突法相比, 该测 量仪消除了膜结构边界对测量结果的影响, 测量结果准确可靠。通过数值仿真与实验结果的对比验证了该装置的正确性, 从理论上解决了膜面张力快速测量的技术问题, 并在实际测量中得到了稳定的结果, 从而为膜面张力测量提供了新方法。

关键词: 顶突法, 膜结构, 膜面张力测量仪

\section{1. 引言}

张拉膜结构由正放雉形双曲面膜单元与县浮桅杆和 下拉索构成, 利用柔性钢索来支承受力结构使膜面张拉形
成稳定的空间曲面。钢索或桅杆可以调整, 以达到设计的 张力值。为保证膜面的稳定性, 张拉膜结构通常以“负高 斯曲面”为基础, 这类曲面由一组张紧的“拱形”单元和与 其反向的“悬挂”单元组成, 这两组元素分别由膜材织物经、 纬两个方向的纤维构成。这种曲面形状有一个重要特点是 
可以在不显著改变曲面形状的前提下就可以对整体曲面 施加预张力。在边界条件一定的前提下, 膜结构的曲面形 状是固定的。如果整体或部分地改变边界条件就会改变整 个膜的形状, 因此, 我们可以认为每个曲面都是特定边界 条件下的产物 [1-2]。

张力膜结构是一种柔性结构体系, 与传统结构不同的 是其刚度由结构的几何曲面和预应力来提供。为了使薄膜 结构具有一定的刚度, 必须对其施加预应力, 如何准确地 测量膜材预张力值一直是业界致力研究的一个方向。预张 力的均匀传递, 膜裁剪亦相对准确, 不但能保证结构的安 全性, 对消除褶皱也是有效手段。根据国内外的一些研究, 目前测量膜材张力的方法主要有：应变法、拉伸法、振动 法、位移法、摄影测量法和“拟索法”等[3]。现有的膜面张 力测量仪或测量的可信度较低、测量误差较大, 或是笨重, 不方面在工程中测量及张力测量方法中。因此, 发明一种 能方便应用于工程实际的膜面张力测量仪器和测试方法 具有重要的理论意义和工程实用价值。

\section{2. 顶突法测膜面张力}

\section{1. 膜材预张力测量仪理论依据: “三点弯曲法”}

在以往的研究中, 被测膜面的测量区域选定为长方形, 当膜材长向短向长度之比为 $3: 1$ 时, 长向预应力对中心点 位移的影响比较小; 当膜材长向短向长度之比大于 5 时, 长向的预张力和弹性模量变化对膜材平面外均布荷载与 平面外位移关系的影响几乎可以忽略不计, 此时的膜材单 向板的特点就已经很明显。由此可知, 就一个膜结构的局 部区域而言, 当长方形膜材区域的长边与短边的长度之比 大于一定的值时, 其他参数保证不变, 就可以认为该区域 在平面外均布荷载作用下的受力性能与单向板类似。在平 面外均布荷载作用下, 单向板中心点的平面外位移与短向 的应力主要决定于板短向的性质和跨度而与长向的参数 关系较小 [4-8]。另外其测量区域达不到四边固定的边界条 件, 导致测量结果有较大的误差。

本课题组通过对以往研究的改进, 采用具有磁性加持 的圆环来固定边界, 可以让膜材在边界上沿膜面自由滑动, 由于边界条件是可以滑动的, 因此集中力不仅引起测量区 域内膜材内力的变化, 同时在测量区域外一定范围内也引 起内力的变化, 很好的解决了四边固定的问题, 并且减少 了实验误差。

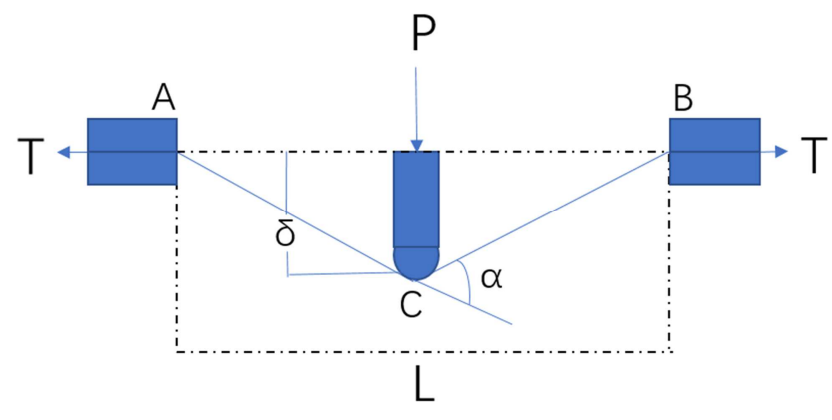

图1 顶突法测量膜面张力工作原理。
压头在 $\mathrm{C}$ 点相对于 $\mathrm{A} 、 \mathrm{~B}$ 点向下压紧过程中, 会在膜面 产生位移信号 $\delta$ 和压紧力信号 $\mathrm{P}$, 设中心线偏折角为 $\alpha$ 。由 公式 $\mathrm{P}=2 \mathrm{~T} \sin (\alpha / 2), \delta=(\mathrm{PL} / 4 \mathrm{~T})$ 可知, 由单一信号 $\mathrm{P}$ 确定 $\mathrm{T}$, 测力器上可确定 $\delta$, 由 $\delta$ 和 $\mathrm{P}$ 可求得张力 $\mathrm{T}[9]$ 。

本次实验中的膜面张力计采用顶突结构, 在垂向施加 一个恒定的载荷, 在同样的膜材条件下, 膜面张力越大, 顶突深度越小。

\section{2. 膜材预张力测量仪}

本课题组设计的新型顶突式膜面张力计由磁性夹持 固定边界底座、圆筒外壳、螺旋压杆、压头, 力传感器和 位移计组成, 如图1所示。在实际膜面结构测量时, 首先 将磁性加持底座上下两部分分别置于膜的上下两面并依 靠强力磁铁紧紧贴合, 分割出一固定边界区域; 张力测量 仪置于膜面固定, 压头在中心位置; 旋转压头, 压杆下压 一定位移量, 读取回弹力或者在压头上施加固定力, 通过 位移计读出压头的顶突深度, 读取顶突位移和回弹力的数 据, 得到一根回弹力一顶突位移的曲线, 该曲线与标定曲 线族比对，换算出膜面的张力 [10]。

该测试方法有两个要点, 一是通过磁夹式固定边界, 测量时膜面的挠度是一固定的三点弯模型, 与实际膜面的 大小和边界条件无关，二是在该测量方法在标定时，对一 定的张力, 膜上的每一个测点均可以得到一条回弹力一顶 突深度曲线, 对于不同的张力, 得到一个曲线族。

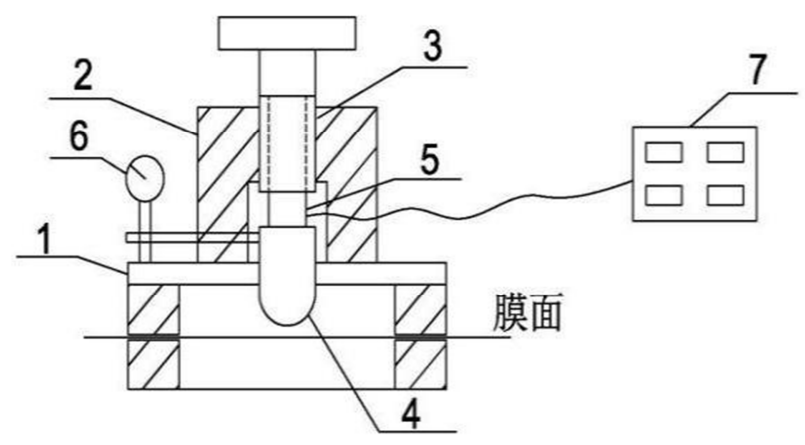

(a)正视图

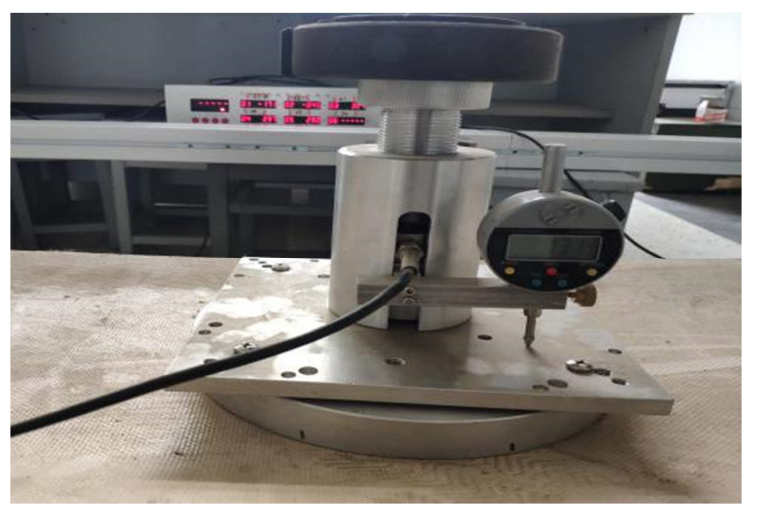

(b)实物图

图2 测试装置示意图（磁性加持固定边界底座（1）、圆筒外壳（2）、 螺旋压杆 (3)、压头 (4)、力传感器 (5) 和位移计 (6), 数显式力 值显示器 (7) )。 


\section{3. 膜材预张力测量仪的标定及实验步骤}

\subsection{1. 膜材预张力测量仪的标定}

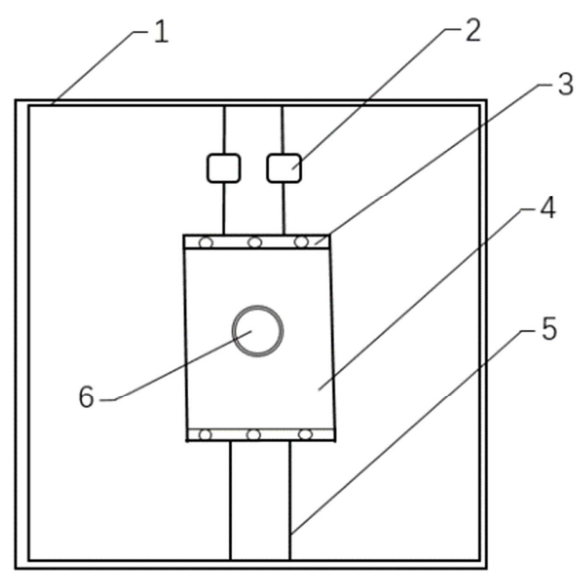

图3 试验台示意图(1-框架 2-拉力传感器 3-膜面夹具 4-膜面 5-链条 6-圆环测试区域)。

本试验中采用的张力计在南京理工大学的试验台上 标定, 标定台上膜试样的安装和标定如图2和图3所示。标 定方法如下:
（1）采用夹持式，制作膜面 $200 \times 700 \mathrm{~mm}$ 的试样，小 试样的张拉点每边两个点。

(2) 对小试样单向张拉, 张拉值用力传感器测量。

（3）将膜标定试样分别张拉到工作载荷 $1000 \mathrm{~N} / \mathrm{m}$ 、 $2000 \mathrm{~N} / \mathrm{m} 、 3000 \mathrm{~N} / \mathrm{m} 、 4000 \mathrm{~N} / \mathrm{m} 、 5000 \mathrm{~N} / \mathrm{m} 、 6000 \mathrm{~N} / \mathrm{m}$ 。

(4) 将张力计放置在膜上, 压头接触膜面, 压头的 位置调零。

（5）在压头上施加 $5 \mathrm{~N} 、 12 \mathrm{~N} 、 15 \mathrm{~N} 、 20 \mathrm{~N}$ 的砝码, 通 过位移计分别读出压头的顶突深度。

(6) 对比各试样上的数值, 进行对比标定。

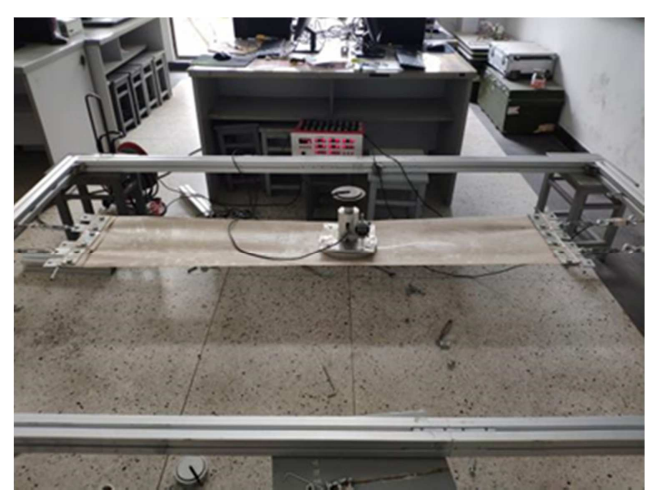

图4 膜面试样标定。

\subsection{2. 不同张力下的标定结果}

表1 不同张力、不同砝码下张力测量仪的测量结果。

\begin{tabular}{llllll}
\hline 编号 & 张力值 $(\mathbf{N} / \mathbf{m})$ & 平均顶突值 $(\mathbf{5 N})$ & 平均顶突值 $(\mathbf{1 2 N})$ & 平均顶突值 $(\mathbf{1 5 N})$ & 平均顶突值 $(\mathbf{2 0 N})$ \\
\hline 1 & 1000 & 2.71 & 3.92 & 6.35 & 7.57 \\
2 & 2000 & 2.52 & 3.52 & 5.24 & 6.86 \\
3 & 3000 & 2.06 & 2.90 & 4.83 & 4.12 \\
4 & 4000 & 1.91 & 2.44 & 3.21 & 3.65 \\
5 & 5000 & 1.63 & 1.99 & 2.57 & 2.74 \\
6 & 6000 & 1.12 & 1.58 & 1.83 & 2.74 \\
\hline
\end{tabular}

由表1可知, 对一定的张力, 膜上的每一个测点均可 以得到一条回弹力一顶突位移曲线, 对于不同的张力, 可 以得到一个曲线族如图4所示, 其中蓝色虚线为实验测量

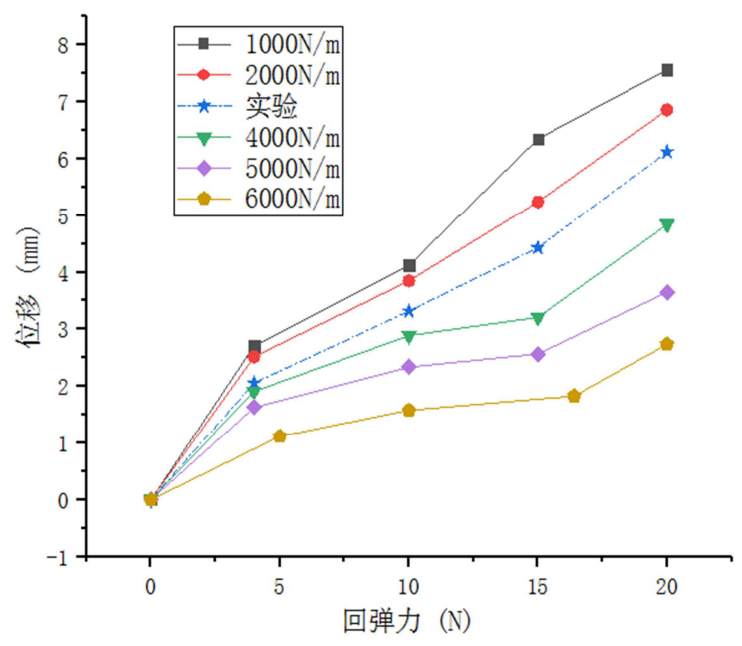

图5 标定曲线族图。
结果, 通过与标定曲线族的对比, 可知实验测得的膜面张 力为 $3000 \mathrm{~N} / \mathrm{m}$ 。

\section{3. 数值仿真对试验结果的验证}

\section{1. 有限元模型的建立}

ABAQUS 是一套功能强大的基于有限元法的工程模 拟软件, 具备十分丰富、可模拟任意实际形状的单元库。 另外还具有各种类型的材料模型库, 可以模拟大多数典型 工程材料的性能，例如金属、橡胶、高分子材料、复合材 料、钢筋混凝土以及膜材等。ABAQUS还可以分析复杂的 固体力学和结构力学系统, 特别是能够驾驭非常庞大的问 题和模拟非线性的影响。

ABAQUS有两个主要的分析模块: ABAQUS/Standard 提供了通用的分析能力, 如应力和变形、热交换、质量传 递等; ABAQUS/Explicit应用对时间进行显式积分的动态 模拟, 提供了应力/变形分析的能力。

此次分析所用的有限元计算软件为ABAQUS, 利用 ABAQUS进行有限元建模时, 最重要的是单元的选取和计 算控制方法的确定。由于膜材本身的特性, 在建立有限元 
模型时, ABAQUS有两种单元形式可供选择: 膜单元和壳 单元。由于膜单元没有弯曲刚度, 只能传递面内应力, 在 计算膜结构单元建立的有限元模型时, 可能会导致结果不 收玫, 因此, 采用壳单元来建立有限元模型。分别建立矩 形膜面、圆环和压头三个模型, 然后再装配在一起进行分 析。压头通过一个半径为 $0.005 \mathrm{~m}$ 的四分之一圆加长度为 $0.003 \mathrm{~m}$ 的线段旋转 $360^{\circ}$ 来建立。

在有限元中, 接触条件是一类特殊的不连续的约束, 它允许力从模型的一部分传递到另一部分。因为只有当两 个表面接触时才用到接触条件, 所以这种约束是不连续的; 当两个接触面分开后, 这种约束便不再存在。因此, 分析 方法必须能够判断什么时候两个表面是接触的并且采用 合适的接触约束。同样的, 该分析方法也能判断什么时候 两个表面是脱离的并解除解除约束。

本次模拟中涉及到压头与膜面的接触问题, 考虑到压 头在向下压的过程为“小应变, 大变形”, 即刚接触时为压 头顶部与膜面的点接触, 随着力的增加, 由点接触变成压 头与膜面的面接触。因此在建立有限元模型时, 将压头顶 部与膜面接触设置为表面与表面接触, 计算有限元模型如 图6-7所示:

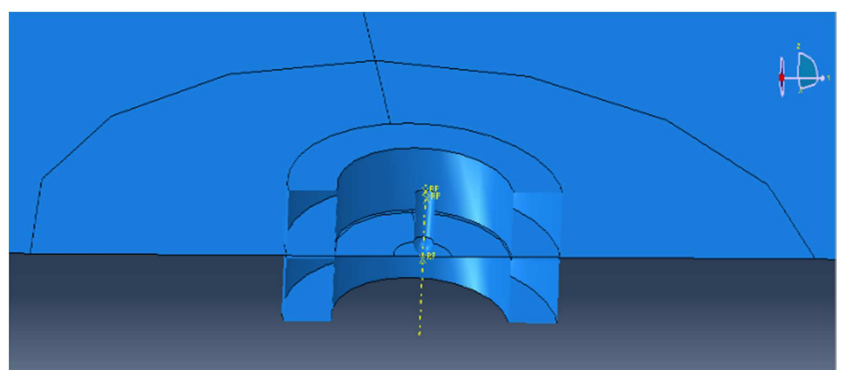

图6 有限元模型剖面图。

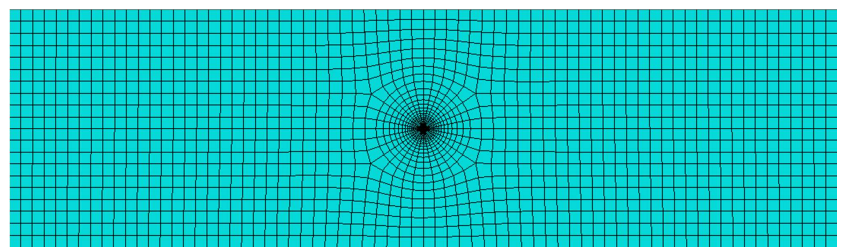

图7 有限元模型网格化。

\section{2. 预张力测量过程的有限元模拟}

选用膜面 $350 \times 1650 \mathrm{~mm}$ 的试样, 来验证预张力测量方 法的可行性。该膜面的参数如下所示: 厚度为 $0.078 \mathrm{~m}$, 假 设正交各向异性膜材的 $\mathrm{x} 、 \mathrm{y}$ 方向平面分别为其经向和纬向, 两个方向的弹性模量分别为 $E_{X}=880 \mathrm{~N} / \mathrm{mm}^{2}$, $E_{\mathrm{y}}=1550 \mathrm{~N} / \mathrm{mm}^{2}$, 泊松比 $v_{x}=v_{y}=0.3, \mathrm{z}$ 方向的弹性模量为 $1215 \mathrm{~N} / \mathrm{mm}^{2}$, 剪切模量 $\mathrm{G}=17.6 \mathrm{~N} / \mathrm{mm}^{2}$ 。膜面左侧为固定端, 右侧提供拉力, 膜材预应力是通过给膜面施加负压强得到 的。模拟结果如下图所示:

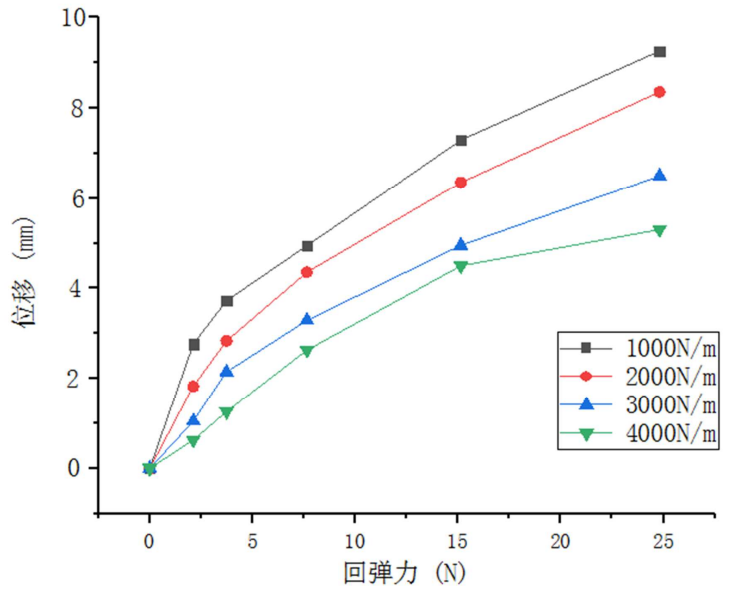

图8 模拟曲线族图。

由上图可知, 在预张力一定的情况下, 随着回弹力的 增加, 顶突位移也会逐渐增加; 在回弹力一定的情况下, 随着预张力的增加, 顶突位移逐渐变小。

\section{3. 模拟结果与实验结果的对比}

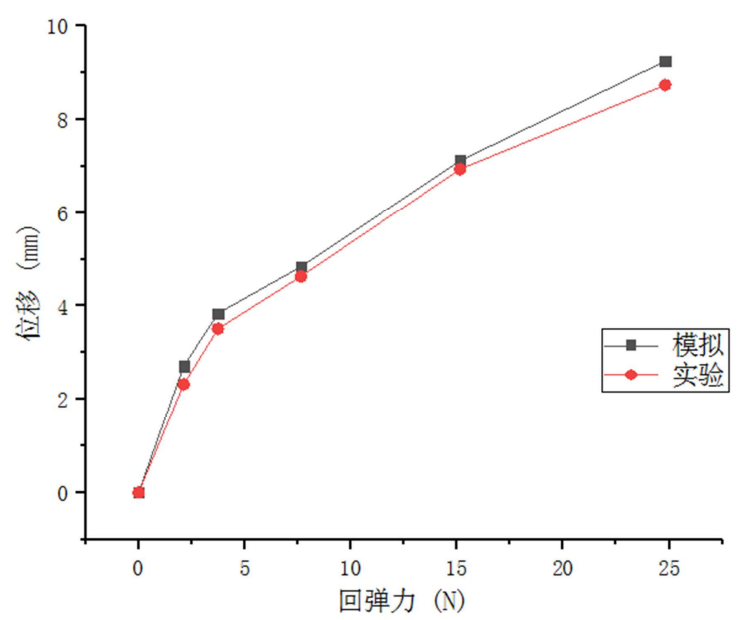

(a) $1000 \mathrm{~N} / \mathrm{m}$

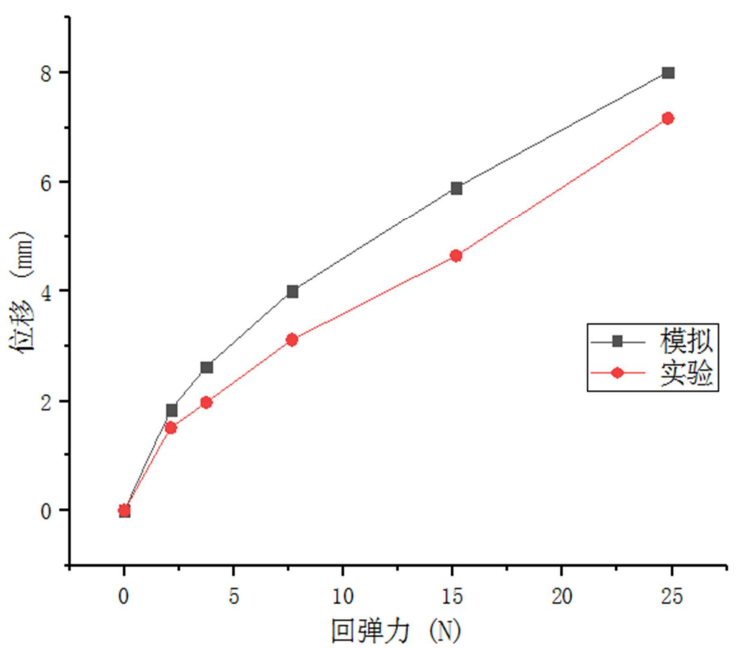

(b) $2000 \mathrm{~N} / \mathrm{m}$ 


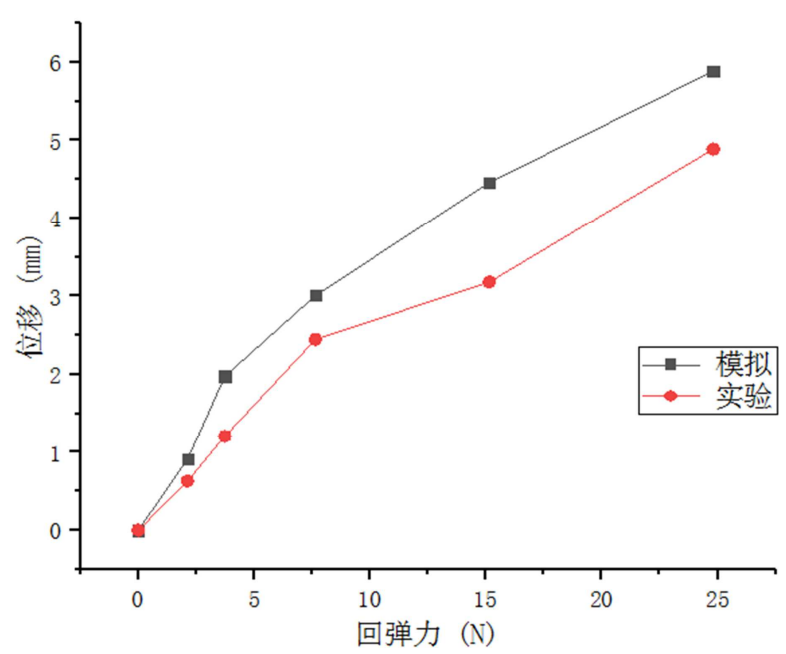

(c) $3000 \mathrm{~N} / \mathrm{m}$

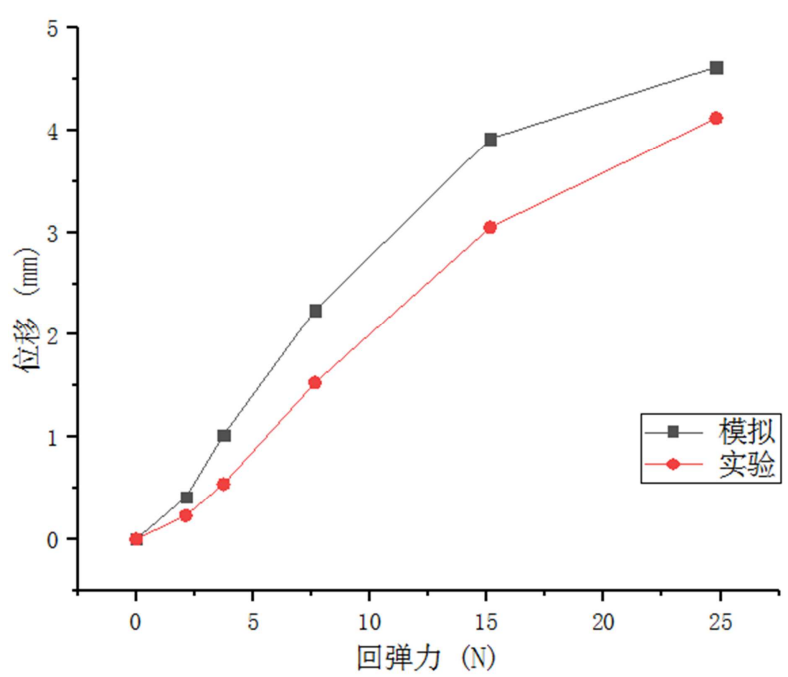

(d) $4000 \mathrm{~N} / \mathrm{m}$

图9 预张力一定, 模拟与实验结果对比。

由上图9的对比可知, 模拟结果与实验结果的回弹力位移曲线的发展规律基本上是一致的。当预张力为 $1000 \mathrm{~N} / \mathrm{m}$ 时, 由于预张力较小, 圆环能够起到固定边界的 作用, 模拟与实验的回弹力-位移曲线接近重合; 随着预 张力的逐渐增大, 在回弹力一定的情况下, 模拟结果的位 移比实验结果的位移大, 这是因为模拟是理想状态, 圆环 内的固定边界与外界无关; 当预张力足够大时, 圆环起不 到固定边界的作用, 所得到的位移不再是圆环两端及压头 作用下的位移, 还需要考虑边界的影响。

\section{4. 结论}

鉴于现有膜材预张力测量方法和设备的精确度较低, 使用不方便, 过于笨重, 应用也不广泛, 设计了一种具有 固定边界的顶突式膜面张力测量仪来方便快捷的测量膜
面预张力。顶突法通过利用磁性加持圆环固定边界, 实现 了边界的缩小, 消除了实际测量中由于边界过大对膜面张 力测量造成的影响, 并减少误差; 同时, 使得顶突位移反弹力只与张力有关, 与实际工程中膜的大小、形状及边 界条件无关, 很好的解决了有些方法仅适用于某些已经标 定的膜材, 要应用于其他种类的膜材则必须另行标定的问 题。另外, 与传统的点标定方法相比, 本实验利用力与位 移的关系来绘制标定曲线族, 使得实验结果更加准确 [11-15]。

\section{参考文献}

[1] 张其林.索和膜结构 [M].上海: 同济大学出版社, 2002 。

[2] 陈务军著.膜结构工程设计 [M]. 北京: 中国建筑工业出版 社,2004。

[3] 钟莉莉.膜结构预张力测试系统的研制[D].国防科学技术大 学, 2007:7 10。

[4] 孙战金, 张其林, 杨宗林.采用数值计算进行膜材预张力测 量方法初探 [J].振动、测试与诊断, 2005，25(1): 31～35。

[5] 孙战金.建筑膜材预张力值测试的理论和实验研究:[D]. 上 海:同济大学土木工程学院, 2005:58 63。

[6] 刘长江. “弹射法”测量膜结构预张力的理论及应用研究[D]. 重庆: 重庆大学土木工程学院, 2009:4 9。

[7] 徐俊豪, 张营营, 赵玉帅, 周神, 张其林. 聚氯乙烯膜材各 向异性超弹性本构模型 [J]. 建筑结构学报, 2019, 40(2):198 205。

[8] 邓长根. 一种简便实用的膜张力测量装置 [J]. 钢结构与建筑 业, 2002，2(4): 50 53。

[9] 缪建成,陈关龙,金华. 小直径柔性钢索预紧张力的测量与计 算 $[J]$.《力学与实践》,2006,28(2):30 31。

[10] www.okok.org. 《结构理论与工程实践》. 中国计划出版 社, 2005:260 265。

[11] 福斯特(英),莫莱尔特(比)著.杨庆山,姜忆南译.欧洲张力膜 结构设计指南[M]. 北京:机械工业出版社,2006。

[12] 陆鉴恒.索膜结构动力松弛法的研究与改进[D]. 武汉:华中 科技大学, $2006: 19 \sim 39$ 。

[13] 蔡文琦. 索膜结构的形态分析设计及静力性能研究 [D].杭州: 浙江大学,2006:58 71。

[14] 罗斌等.建筑织物膜材的单轴和双轴拉伸材性试验研究 [J]. 建筑结构,2007,37(7):104-106,61。

[15] Oska, Membrane Structures [M]. Vol-1.Taiyo kogyo Corp. Japan, 1991。 